\title{
A robust adaptive tracking controller for an aircraft with uncertain dynamical terms *
}

\author{
Ilker Tanyer* Enver Tatlicioglu* Erkan Zergeroglu ${ }^{* *}$ \\ * Department of Electrical $\&$ Electronics Engineering, Izmir Institute \\ of Technology, Izmir, 35430 Turkey, (e-mails: \\ [ilkertanyer,enver]@iyte.edu.tr). \\ ** Department of Computer Engineering, Gebze Institute of \\ Technology, 41400, Gebze, Kocaeli, Turkey, (e-mail: \\ ezerger@bilmuh.gyte.edu.tr)
}

\begin{abstract}
This work presents, the design and the corresponding analysis of a nonlinear controller for an aircraft system subject to uncertainties in the dynamics and additive state-dependent nonlinear disturbance-like terms. Specifically; dynamic inversion technique in conjunction with a robust integral of the signum of the error feedback and an adaptive term is utilized in the overall controller design. Lyapunov based stability analysis techniques are then utilized to prove global asymptotic convergence of the tracking error.
\end{abstract}

Keywords: Robust adaptive control; Uncertain dynamic systems; Aircrafts; Asymptotic stability;

\section{INTRODUCTION}

Dynamic inversion (DI) is a control design approach for nonlinear systems [1], [2] where the main idea is to transform the nonlinear system into a linear time-invariant system via applying a change of variables. The controller design is then carried out to drive the transformed linear dynamics to a reference model as in [3], [4] and [5].

The DI technique is generally preferred when system dynamics is known. However, as commonly stated by control engineering community, no model is exact and lack of accurate knowledge of model may have undesired effects. For example, the uncertainties in the input matrix can result in an increase in the inversion error and DI based algorithms can have difficulty compensating this.

Some past research was devoted to fusing robustifying terms with DI technique. Liu et al. proposed a robust DI method based on sliding mode control for attitude tracking of an aerial vehicle [6]. In [7], Yamasaki et al. proposed a robust DI controller for trajectory tracking control of an unmanned aerial vehicle (UAV). A robust nonlinear controller combined with DI technique was applied to a nonlinear aircraft model in [8]. In [9], Xie et al. proposed a DI method fused with a proportional integral controller to linearize the dynamics of UAVs. Adaptive control techniques were also combined with DI to compensate for structured uncertainties. In [10], a DI based adaptive controller was developed for attitude tracking of X-33 space plane. In [11], an adaptive DI based switching control methodology was developed for compensating structured uncertainties. Liu et al. used DI in conjunction with a nonlinear model reference adaptive controller (MRAC)

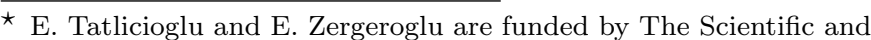
Technological Research Council of Turkey via grant number 113E147. All the correspondence should be addressed to E. Tatlicioglu.
}

based on neural networks [12]. In [13], an adaptive dynamic inversion (ADI) controller was developed for feedback linearization control of a flexible spacecraft. Wang and Stengel designed an ADI controller for a miniature UAV [14]. Calise and Rysdek developed an ADI controller which was a feedback linearizing controller in combination with adaptive feedforward neural network terms [15]. Lavretsky and Hovakimyan designed a direct MRAC augmented with a DI controller and compensated structured uncertainties [16]. In most of the works cited above, ADI controllers successfully compensated structured uncertainties, but failed to compensate unstructured uncertainties.

In DI controller literature, there are also some past works aimed designing controllers to compensate for both structured and unstructured uncertainties. To name a few, in [17], [18], [19], [20], [21], neural networks were fused with ADI based controllers where boundedness of the tracking error was ensured, however the aforementioned studies were unable to prove asymptotic tracking. In [22] and [23], Shin et al. developed a tracking controller by fusing robust integral of the signum of the error (RISE) feedback with neural network feedforward terms for a rotorcraft-based UAV and guaranteed semi-global asymptotic tracking. In [24], MacKunis et al. combined the robust controller in [25] with a DI technique to obtain asymptotic output tracking for aircraft systems with an uncertain input matrix and subject to additive disturbances. While exponential tracking was obtained main drawback of the proposed controller was that the signum of the time derivative of the output was required (i.e., signs of the acceleration values were needed).

Though some aircraft systems are equipped with accelerometers and therefore acceleration measurements are available on these devices, utilizing acceleration measurements in controller design is not preferred. The main 
reason is due to the sensor related issues, such as calibration and possible sensor failures (which may result in inaccurate measurements or disruption), that can produce significant stability problems. Also, in some cases, using accelerometers may not be feasible due to the extra cost. It should also be noted that using an additional sensor complicates the overall sensing system. Best way to avoid these problems is, if possible, not to use acceleration information in the control design. This is one of the motivations of our work.

In this paper, asymptotic tracking control of an uncertain aircraft subject to additive disturbances is discussed. The state and the input matrices are considered to be uncertain, and the system dynamics is subject to an additive state-dependent disturbance. Only the output of the aircraft is considered to be available for the control design, thus the acceleration information is not needed. In the error system development, a matrix decomposition is utilized to cope with the possibly sign indefinite and not necessarily symmetric matrix that the control input is multiplied with. In the controller design, an adaptive term fused with a modification of the RISE feedback in [25], [26] is utilized. The proposed controller ensures global asymptotic stability of the tracking error via the use of novel Lyapunov based design and analysis techniques.

\section{AIRCRAFT MODEL}

In this paper, similar to [3], [10], [27] and [28], the following commonly utilized nonlinear state space model of an aircraft system is considered

$$
\dot{x}=A x+f(x, t)+B u, y=C x
$$

where $x(t) \in \mathbb{R}^{n}$ denotes the state vector, $A \in \mathbb{R}^{n \times n}$ is the constant state matrix, $f(x, t) \in \mathbb{R}^{n}$ is a state-dependent nonlinear disturbance-like term (including gravity, inertial coupling and nonlinear gust modeling effects), $B \in \mathbb{R}^{n \times m}$ is the constant input matrix, $u(t) \in \mathbb{R}^{m}$ is the control input, $C \in \mathbb{R}^{m \times n}$ is the known output matrix, and $y(t) \in$ $\mathbb{R}^{m}$ is the output. The number of states is assumed to be greater than the number of outputs (i.e., $n>m$ ). The subsequent development requires the aircraft model in (1) to satisfy the following properties.

Assumption 1. The system in (1) is controllable.

Assumption 2. The nonlinear disturbance-like term $f(x, t)$ is considered to be equal to the sum of state-dependent uncertainties, denoted by $f_{1}(x) \in \mathbb{R}^{n}$, and time-dependent uncertainties, denoted by $f_{2}(t) \in \mathbb{R}^{n}$. The time-dependent uncertainty vector $f_{2}(t)$ is continuously differentiable and bounded up to its first order time derivative, and the statedependent uncertainty vector $f_{1}(x)$ depends on the state vector $x(t)$ via trigonometric and/or bounded arguments only and thus it is assumed that $f_{1}(x)$ and $\partial f_{1}(x) / \partial x$ are bounded for all $x(t)$ (see [29] for the precedence of this type of assumption).

\section{CONTROL DESIGN}

Aside from the boundedness objective (i.e., all the signals are required to remain bounded under the closed-loop operation), the main control objective is to design a control law to ensure that the output of the aircraft $y(t)$ tracks the output of the following reference model

$$
\dot{x}_{m}=A_{m} x_{m}+B_{m} u_{m}, y_{m}=C x_{m}
$$

where $x_{m}(t) \in \mathbb{R}^{n}$ is the reference state vector, $A_{m} \in$ $\mathbb{R}^{n \times n}$ is the reference state matrix, $B_{m} \in \mathbb{R}^{n \times m}$ is the reference input matrix, $u_{m}(t) \in \mathbb{R}^{m}$ is the reference input, $C$ is the same output matrix introduced in (1), and $y_{m}(t) \in$ $\mathbb{R}^{m}$ is the reference output. To ensure the stability of the reference model signals, the reference state matrix $A_{m}$ is chosen to be Hurwitz, and the reference input $u_{m}(t)$ and its time derivative are designed as bounded functions of time. Provided these, linear analysis tools can then be utilized to prove that $x_{m}(t), \dot{x}_{m}(t), \ddot{x}_{m}(t)$ and thus, $y_{m}(t), \dot{y}_{m}(t)$, $\ddot{y}_{m}(t)$ are bounded functions of time.

In the subsequent development, the dynamic terms $A, B$, and $f(x, t)$ are considered to be uncertain $^{1}$, and thus, will not be used in the control design. The subsequent control development is derived based on the restriction that only the output $y(t)$ is available (i.e., output feedback controller).

The output tracking error $e(t) \in \mathbb{R}^{m}$ is defined as

$$
e \triangleq y-y_{m}=C\left(x-x_{m}\right)
$$

where (1) and (2) were utilized to obtain the second equality. From (3), it is clear that when $\|e(t)\| \rightarrow 0$ the output of the aircraft tracks the output of the reference model. To simplify the analysis, an auxiliary error signal $r(t) \in \mathbb{R}^{m}$ is introduced to have the following form

$$
r \triangleq \dot{e}+\Lambda e
$$

where $\Lambda \in \mathbb{R}^{m \times m}$ is a constant, positive definite, diagonal control gain matrix. It is noted that due to the time derivative of the output $y(t)$ in $\dot{e}(t), r(t)$ is not available for control design.

Assumption 3. Since the number of states is greater than the number of outputs, there may be some states that cannot be observed through the output. To obtain bounds for the unobservable states, it is considered that the state vector can be partitioned as $x=x_{o}+x_{u}$ where $x_{o}(t), x_{u}(t) \in \mathbb{R}^{n}$ contains the observable states and the unobservable states, respectively. Furthermore, the unobservable states are assumed to be partitioned as $x_{u}=x_{u \rho}+x_{u \xi}$ where $x_{u \rho}(t), x_{u \xi}(t) \in \mathbb{R}^{n}$ contain the unobservable states that can be bounded by a function of error signals and a constant, respectively. These bounds are quantified as

$$
\left\|x_{u \rho}(t)\right\| \leq c_{1}\|z\| \text { and }\left\|x_{u \xi}(t)\right\| \leq \xi_{x_{u}}
$$

where $c_{1}, \xi_{x_{u}} \in \mathbb{R}$ are known positive bounding constants and $z \triangleq\left[e^{T}, r^{T}\right]^{T} \in \mathbb{R}^{2 m}$ is the combined error signal. A similar upper bound is assumed for the components of $\dot{x}_{u}(t)$ as well

$$
\left\|\dot{x}_{u \rho}(t)\right\| \leq c_{2}\|z\| \text { and }\left\|\dot{x}_{u \xi}(t)\right\| \leq \xi_{\dot{x}_{u}}
$$

where $c_{2}, \xi_{\dot{x}_{u}} \in \mathbb{R}$ are known positive bounding constants.

Similarly, the reference model state $x_{m}(t)$ is assumed to be partitioned as $x_{m}=x_{m o}+x_{m u}$ where $x_{m o}(t), x_{m u}(t) \in \mathbb{R}^{n}$ contain the entries of the reference state corresponding to the observable states and the unobservable states, respectively. The reader is referred to [29] for the precedence of this assumption.

From (4), following expression can be obtained

$$
r=C A x+\Omega u+C f-C A_{m} x_{m}-C B_{m} u_{m}+\Lambda e
$$

1 This is a direct result of the varying nature of aircraft dynamics due to the operating conditions. 
where (1)-(3) were utilized, and $\Omega \triangleq C B \in \mathbb{R}^{m \times m}$ is an auxiliary constant matrix. It is highlighted that since $B$ is uncertain, then $\Omega$ is uncertain as well. Furthermore, neither symmetry nor positive definiteness of $\Omega$ are unknown. Given these restrictions, the SDU decomposition in [30] is applied to $\Omega$ as

$$
\Omega=S D U
$$

where $S \in \mathbb{R}^{m \times m}$ is a symmetric positive definite matrix, $D \in \mathbb{R}^{m \times m}$ is a diagonal matrix with entries \pm 1 , and $U \in \mathbb{R}^{m \times m}$ is a unity upper triangular matrix. The reader is referred to [31] for the details of the SDU decomposition. Remark 1. The SDU decomposition of $\Omega$ for different aircraft models in the literature [32], [33], [34] resulted in the diagonal matrix $D$ being equal to an identity matrix for all these models. However, for the completeness of the presentation, the subsequent controller will be designed to be applicable to any aircraft model without imposing any restrictions on $D$.

After utilizing the SDU decomposition in (8), the time derivative of (7) is obtained as

$$
\dot{r}=C A \dot{x}+S D U \dot{u}+C \dot{f}-C A_{m} \dot{x}_{m}-C B_{m} \dot{u}_{m}+\Lambda \dot{e} .
$$

Since $S$, introduced in (8), is symmetric and positive definite, then so is its inverse, defined as $M \triangleq S^{-1} \in$ $\mathbb{R}^{m \times m}$. Premultiplying (9) with $M$ yields

$$
M \dot{r}=-e+D U \dot{u}+N .
$$

where $N(x, \dot{x}, t) \in \mathbb{R}^{m}$ is defined as

$$
N \triangleq M\left[C A \dot{x}+C \dot{f}-C A_{m} \dot{x}_{m}-C B_{m} \dot{u}_{m}+\Lambda \dot{e}\right]+e .
$$

The auxiliary signal $N(\cdot)$ can be partitioned as

$$
N=N_{d}+N_{L P}+\widetilde{N}
$$

where $N_{d}(t), N_{L P}(t), \tilde{N}(x, \dot{x}, e, r) \in \mathbb{R}^{m \times 1}$ are defined as

$$
\begin{aligned}
N_{d} \triangleq & M C\left[\left(A+\frac{\partial f_{1}(x)}{\partial x}\right) \dot{x}_{u \xi}+\left(\frac{\partial f_{1}(x)}{\partial x} \dot{x}_{m o}+\dot{f}_{2}\right)\right] \\
N_{L P} \triangleq & M C\left[-B_{m} \dot{u}_{m}+\left(A-A_{m}\right) \dot{x}_{m o}-A_{m} \dot{x}_{m u}\right] \\
\widetilde{N} \triangleq & M C\left(A+\frac{\partial f_{1}(x)}{\partial x}\right)\left[\left(\dot{x}_{o}-\dot{x}_{m o}\right)+\dot{x}_{u \rho}\right] \\
& +M \Lambda(r-\Lambda e)+e .
\end{aligned}
$$

Remark 2. In the above partitioning, the uncertain terms that could be bounded with constant were grouped into $N_{d}(t)$, the terms that can be linearly parameterized were grouped into $N_{L P}(t)$, and the terms that could be bounded by functions of error signals were grouped into $\tilde{N}(t)$.

From Assumptions 2 and 3, and the boundedness of the reference model signals, it can be shown that $N_{d}(t)$ is a bounded function of time in the sense that $\left\|N_{d}(t)\right\| \leq \zeta_{N_{d}}$ $\forall t$ where $\zeta_{N_{d}} \in \mathbb{R}$ is a positive bounding constant (or alternatively, $\left|N_{d, i}\right| \leq \zeta_{N_{d, i}} \forall t$ with $\zeta_{N_{d, i}} \in \mathbb{R}$ being positive bounding constants).

It is straightforward to show that the auxiliary term $N_{L P}(t)$ defined in (14) can be written as a multiplication of a known regression matrix and an uncertain constant parameter vector. On the other hand, the auxiliary errorlike term $\widetilde{N}(t)$ can be upper bounded as

$$
\|\widetilde{N}(t)\| \leq \rho\|z(t)\|
$$

where $\rho \in \mathbb{R}$ is a positive bounding constant.
Based on the subsequent stability analysis, the control input is designed as

$$
\begin{aligned}
u= & -D K\left[e(t)-e(0)+\Lambda \int_{0}^{t} e(\sigma) d \sigma\right]-D \Pi \\
& -D \int_{0}^{t} Y(\sigma) \hat{\phi}(\sigma) d \sigma
\end{aligned}
$$

where $\Pi(t) \in \mathbb{R}^{m}$ is an auxiliary filter signal updated according to ${ }^{2}$

$$
\dot{\Pi}(t)=\beta \operatorname{Sgn}(e(t)) \text { with } \Pi(0)=0_{m \times 1}
$$

and $\beta \in \mathbb{R}^{m \times m}$ is a constant, positive definite, diagonal, control gain matrix, $\operatorname{Sgn}(\cdot)$ denotes the vector signum function, $K \in \mathbb{R}^{m \times m}$ is a constant, positive definite, diagonal, control gain matrix and is designed as

$$
K=I_{m}+k_{g} I_{m}+\operatorname{diag}\left\{k_{d, 1}, k_{d, 2}, \ldots, k_{d, m-1}, 0\right\}
$$

with $k_{g}, k_{d, 1}, \ldots, k_{d, m-1} \in \mathbb{R}$ being positive gains. In (17), $Y(t) \in \mathbb{R}^{m \times p}$ denotes the regression matrix which is composed of reference signal, its time derivatives, and other known quantities, and is defined from

$$
Y \phi=N_{L P}-D\left(U-I_{m}\right) D Y \hat{\phi} \text {. }
$$

where $\phi \in \mathbb{R}^{p \times 1}$ is the unknown parameter vector with its estimate $\hat{\phi}(t) \in \mathbb{R}^{p \times 1}$ designed as

$$
\begin{aligned}
\hat{\phi}= & \operatorname{Proj}\left(\Gamma \left(Y^{T} e-\int_{0}^{t} \frac{d Y^{T}(\sigma)}{d \sigma} e(\sigma) d \sigma\right.\right. \\
& \left.\left.+\int_{0}^{t} Y^{T}(\sigma) \Lambda e(\sigma) d \sigma\right)\right) .
\end{aligned}
$$

In (21), the term $\operatorname{Proj}($.$) is the projection operator that$ ensures the boundedness of the parameter estimate vector and its time derivative. At this point, we want to point out that since $U$ is unity upper triangular then $U-I_{m}$ is strictly upper triangular and thus there is no algebraic loop in finding $Y(t)$ from (20). The time derivative of (21) yields

$$
\dot{\hat{\phi}}=\operatorname{Proj}\left(\Gamma Y^{T} r\right)
$$

where (4) was utilized. The time derivative of the control input in (17) is obtained as

$$
\dot{u}=-D K r-D \beta \operatorname{Sgn}(e)-D Y \hat{\phi}
$$

where (4) and (18) were utilized. After substituting (23) into (10), following closed-loop error system is obtained

$$
\begin{aligned}
M \dot{r}= & \widetilde{N}+N_{d}-e-D\left(U-I_{m}\right) D K r-K r \\
& -D U D \beta \operatorname{Sgn}(e)+Y \widetilde{\phi}
\end{aligned}
$$

where $(12)$ and (20) were utilized and $\widetilde{\phi}(t) \triangleq \phi-\hat{\phi} \in \mathbb{R}^{p \times 1}$ is the parameter estimation error.

The term $D\left(U-I_{m}\right) D K r$ can be rewritten as

$$
D\left(U-I_{m}\right) D K r=\left[\Phi^{T}, 0\right]^{T}
$$

where the entries of $\Phi(r) \in \mathbb{R}^{(m-1) \times 1}$ are defined as

$$
\Phi_{i}=d_{i} \sum_{j=i+1}^{m} d_{j} k_{j} U_{i, j} r_{j} \text { for } i=1, \ldots,(m-1) .
$$

2 Throughout the paper, $I_{n}$ and $0_{m \times r}$ will be used to represent an $n \times n$ standard identity matrix and an $m \times r$ zero matrix, respectively. 
Since $d_{i}= \pm 1 \forall i=1, . ., m$, for the entries of $\Phi$, following upper bound can be written

$$
\left|\Phi_{i}\right| \leq \sum_{j=i+1}^{m} k_{j} \zeta_{U_{i, j}}\left|r_{j}\right| \leq \zeta_{\Phi_{i}}\|z\|
$$

where $\zeta_{U_{i, j}}$ are positive bounding constants satisfying $\zeta_{U_{i, j}} \geq\left|U_{i, j}\right| \forall i, j$. It is important to highlight that $\zeta_{\Phi_{i}}$ depends on the control gains $k_{i+1}, \ldots, k_{m}$.

\section{STABILITY ANALYSIS}

We are now ready to state the following theorem;

Theorem 1. The robust adaptive controller in (17), (18), (21) ensures global asymptotic tracking in the sense that

$$
\|e(t)\| \rightarrow 0 \text { as } t \rightarrow \infty
$$

provided that the control gain matrices $K$ and $\beta$ are selected by using the following procedure:

(1) For $i=m, \beta_{m}$ is selected according to

$$
\beta_{m} \geq \zeta_{N_{d, m}}\left(1+\frac{\gamma_{2}}{\Lambda_{m}}\right)
$$

and from $i=m-1$ to $i=1, \beta_{i}$ are selected according to

$$
\beta_{i} \geq\left(\zeta_{N_{d, i}}+\sum_{j=i+1}^{m} \zeta_{\Psi_{j}} \beta_{j}\right)\left(1+\frac{\gamma_{2}}{\Lambda_{i}}\right)
$$

where $\gamma_{2} \in \mathbb{R}$ is some positive bounding constant and the subscript $i=1, \ldots, m$ denotes the $i$-th element of the vector or the diagonal matrix,

(2) Control gain $k_{g}$ is chosen big enough to decrease the constant $\frac{\rho^{2}}{4 k_{g}}$,

(3) Control gains $k_{d, i}, i=1, \ldots,(m-1)$ are chosen big enough to decrease the constant $\sum_{i=1}^{m-1} \frac{\zeta_{\Phi_{i}^{2}}}{4 k_{d, i}}$.

Proof. The proof of theorem consists of four subproofs. Firstly, in Appendix A, boundedness of all the signals under the closed-loop operation will be presented. Secondly, in Appendix B, we present a supporting lemma and its proof. The proof of this lemma provides us to form an upper bound on the terms $\int_{0}^{t}\left|\dot{e}_{i}(\sigma)\right| d \sigma$, which will then be utilized in the next part of the proof. In Appendix $\mathrm{C}$, the positiveness of an auxiliary integral term will be demonstrated. Finally, in Appendix D, the asymptotic convergence of the output tracking error is proven.

\section{CONCLUSION}

In this work, we present the design of a nonlinear robust adaptive controller for aircrafts subject to uncertainties in the dynamics and additive state-dependent nonlinear disturbance-like terms. In the design of the controller, DI technique was fused with an adaptive term to compensate for structured uncertainties and with the robust integral of the signum of the error feedback term to compensate for unstructured uncertainties. Novel Lyapunov type stability analysis techniques were utilized to ensure global asymptotic tracking of the output of a reference model.

\section{Appendix A. BOUNDEDNESS PROOF}

In this appendix, the boundedness of all the signals under the closed-loop operation will be demonstrated. Let $V_{1}(z) \in \mathbb{R}$ be a Lyapunov function defined as

$$
V_{1} \triangleq \frac{1}{2} e^{T} e+\frac{1}{2} r^{T} M r
$$

which can be upper and lower bounded as

$$
\frac{1}{2} \min \left\{1, M_{\min }\right\}\|z\|^{2} \leq V_{1}(z) \leq \frac{1}{2} \max \left\{1, M_{\max }\right\}\|z\|^{2}
$$

where $M_{\min }$ and $M_{\max }$ denote minimum and maximum eigenvalues of $M$, respectively. Time derivative of the Lyapunov function is obtained as

$$
\begin{aligned}
\dot{V}_{1}= & e^{T}(r-\Lambda e)+r^{T}\left[N_{d}+\widetilde{N}-e-D\left(U-I_{m}\right) D K r\right. \\
& -K r-D U D \beta \operatorname{Sgn}(e)+Y \widetilde{\phi}]
\end{aligned}
$$

where (4) and (24) were substituted. After utilizing (27), following upper bound can be obtained

$$
r^{T}\left[\Phi^{T}, 0\right]^{T}=\sum_{i=1}^{m-1} r_{i} \Phi_{i} \leq \sum_{i=1}^{m-1} \zeta_{\Phi_{i}}\left|r_{i}\right|\|z\| .
$$

As a property of the projection operator, following upper bound can be obtained

$$
r^{T} Y \widetilde{\phi} \leq \zeta_{2}\|r\|
$$

After substituting the upper bounds in Remark 2, and utilizing (A.4) and (A.5), following expression can be obtained

$$
\begin{aligned}
\dot{V}_{1} & \leq-e^{T} \Lambda e+\zeta_{N_{d}}\|r\|+\rho\|r\|\|z\|+\sum_{i=1}^{m-1} \zeta_{\Phi_{i}}\left|r_{i}\right|\|z\| \\
& -r^{T} r-k_{g} r^{T} r-\sum_{i=1}^{m-1} k_{d, i}\left|r_{i}\right|^{2}+\left(\zeta_{1}+\zeta_{2}\right)\|r\|
\end{aligned}
$$

where $r^{T} D U D \beta \operatorname{Sgn}(e) \leq \zeta_{1}\|r\|$ was utilized with $\zeta_{1} \in$ $\mathbb{R}$ being a positive bounding constant. After utilizing following manipulations

$$
\begin{array}{r}
\left(\zeta_{1}+\zeta_{2}+\zeta_{N_{d}}\right)\|r\| \leq \frac{1}{4 \delta}\|r\|^{2}+\delta\left(\zeta_{1}+\zeta_{2}+\zeta_{N_{d}}\right)^{2}(\mathrm{~A} .7) \\
\rho\|r\|\|z\|-k_{g}\|r\|^{2} \leq \frac{\rho^{2}}{4 k_{g}}\|z\|^{2}(\mathrm{~A} .8) \\
\zeta_{\Phi_{i}}\left|r_{i}\right|\|z\|-k_{d, i} r_{i}^{2} \leq \frac{\zeta_{\Phi_{i}}^{2}}{4 k_{d, i}}\|z\|^{2}(\mathrm{~A} .9)
\end{array}
$$

$\forall i=1, \ldots,(m-1)$, where $\delta \in \mathbb{R}$ is a positive damping constant, the right-hand side of (A.6) can be bounded as

$$
\begin{aligned}
\dot{V}_{1} \leq & -e^{T} \Lambda e-r^{T} r+\frac{1}{4 \delta}\|r\|^{2}+\delta\left(\zeta_{1}+\zeta_{2}+\zeta_{N_{d}}\right)^{2} \\
& +\frac{\rho^{2}}{4 k_{g}}\|z\|^{2}+\sum_{i=1}^{m-1} \frac{\zeta_{\Phi_{i}}^{2}}{4 k_{d, i}}\|z\|^{2} .
\end{aligned}
$$

If the control gains $\Lambda, k_{g}, k_{d, 1}, \ldots, k_{d, m-1}$ are selected sufficiently high, following expression can be obtained for the time derivative of the Lyapunov function

$$
\dot{V}_{1} \leq-c_{1} V_{1}+c_{2}
$$

where $c_{1}$ and $c_{2}$ are some positive bounding constants. From (A.11), it is clear that $V_{1}(t) \in L_{\infty}$, and thus, $e(t)$, $r(t) \in L_{\infty}$. From (4), it can be proven that $\dot{e}(t) \in L_{\infty}$. By using (3) and its time derivative, along with the boundedness of the reference model signals, it can be proven that $y(t), \dot{y}(t), x(t), \dot{x}(t) \in \mathcal{L}_{\infty}$. The above boundedness statements and Assumption 2 can be utilized along with 
(1) to prove that $u(t) \in \mathcal{L}_{\infty}$. From (23), it is clear that $\dot{u}(t) \in \mathcal{L}_{\infty}$. After utilizing the above boundedness statements, Assumption 2, and the boundedness of the reference model signals along with (9), it can be proven that $\dot{r}(t) \in \mathcal{L}_{\infty}$. Standard signal chasing algorithms can be used to prove that all the remaining signals are bounded.

\section{Appendix B. LEMMA 1 AND ITS PROOF}

Lemma 1. Provided that $e(t)$ and $\dot{e}(t)$ are bounded, the following expression can be obtained [35]

$$
\int_{t_{0}}^{t}\left|\dot{e}_{i}(\sigma)\right| d \sigma \leq \gamma_{1}+\gamma_{2} \int_{t_{0}}^{t}\left|e_{i}(\sigma)\right| d \sigma+\left|e_{i}\right|
$$

where $\gamma_{1}, \gamma_{2} \in \mathbb{R}$ are some positive bounding constants.

Proof. First, it is noted that if $e_{i}(t) \equiv 0$ on some interval, then $\dot{e}_{i}(t) \equiv 0$ on the same interval, and the inequality (B.1) yields this qualification. Therefore, without loss of generality, the case that $e_{i}(t)$ is absolutely greater than zero on the interval of $\left[t_{0}, t\right]$ is considered. Let $T \in\left[t_{0}, t\right)$ be the last instant of time when $\dot{e}_{i}(t)$ changes sign. Then, on the interval $[T, t], \dot{e}_{i}(t)$ has a constant sign, and hence

$$
\int_{T}^{t}\left|\dot{e}_{i}(\sigma)\right| d \sigma=\left|\int_{T}^{t} \dot{e}_{i}(\sigma) d \sigma\right|=\left|e_{i}(t)-e_{i}(T)\right| .
$$

From the boundedness of $\dot{e}_{i}(t)$, it follows that there exists a constant $\gamma>0$ such that $\left|\dot{e}_{i}(t)\right| \leq \gamma$, therefore

$$
\int_{t_{0}}^{T}\left|\dot{e}_{i}(\sigma)\right| d \sigma \leq \gamma\left(T-t_{0}\right)
$$

By applying the Mean Value Theorem [36], we can obtain the following expression

$$
\int_{t_{0}}^{T}\left|e_{i}(\sigma)\right| d \sigma=\left(T-t_{0}\right) e_{i *} .
$$

where $e_{i *}>0$ is some intermediate value of $\left|e_{i}(t)\right|$ on the interval $\left[t_{0}, T\right]$. From (B.3) and (B.4), following expression can be stated

$$
\int_{t_{0}}^{T}\left|\dot{e}_{i}(\sigma)\right| d \sigma \leq \gamma_{2} \int_{t_{0}}^{T}\left|e_{i}(\sigma)\right| d \sigma
$$

where $\gamma_{2} \triangleq \frac{\gamma}{e_{i *}}$. Combining the relationships in (B.2) and (B.5) yields in (B.1) with $\gamma_{1} \triangleq \sup \left|e_{i}(T)\right|$.

\section{Appendix C. LEMMA 2 AND ITS PROOF}

Lemma 2. Let auxiliary function $L(t) \in \mathbb{R}$ be defined as

$$
L \triangleq r^{T}\left[N_{d}-D U D \beta \operatorname{Sgn}(e)\right]
$$

If the entries of $\beta$ are selected to satisfy (29) and (30), then the auxiliary function $P(t) \in \mathbb{R}$ defined as

$$
P \triangleq \zeta_{b}-\int_{0}^{t} L(\sigma) d \sigma
$$

is nonnegative where $\zeta_{b} \in \mathbb{R}$ is a positive bounding constant.

Proof. The proof can be found in [37].

\section{Appendix D. ASYMPTOTIC STABILITY PROOF}

In this appendix, the asymptotic stability of the output tracking error is presented. Let $V_{2}(w) \in \mathbb{R}$ be a Lyapunov function defined as

$$
V_{2} \triangleq V_{1}+\frac{1}{2} \widetilde{\phi}^{T} \Gamma^{-1} \widetilde{\phi}+P
$$

where $w(t) \triangleq\left[\begin{array}{llll}e^{T} & r^{T} & \widetilde{\phi}^{T} & \sqrt{P}\end{array}\right]^{T} \in \mathbb{R}^{(2 m+p+1) \times 1}$. Nonnegativeness of $P(t)$ was proven in Appendix $\mathrm{C}$ and this validates $V_{2}(w)$ as a Lyapunov function. The Lyapunov function in (D.1) can be upper and lower bounded as

$$
\kappa_{\min }\|w\|^{2} \leq V_{2}(w) \leq \kappa_{\max }\|w\|^{2}
$$

where $\kappa_{\min }, \kappa_{\max } \in \mathbb{R}$ are positive bounding constants defined as

$$
\begin{aligned}
\kappa_{\min } & \triangleq \frac{1}{2} \min \left\{1, M_{\min }, \frac{1}{\Gamma_{\max }}\right\} \\
\kappa_{\max } & \triangleq \max \left\{\frac{1}{2} M_{\max }, 1, \frac{1}{2 \Gamma_{\min }}\right\}
\end{aligned}
$$

with $\Gamma_{\max }$ and $\Gamma_{\min }$ denoting maximum and minimum eigenvalues of $\Gamma$, respectively. Taking the time derivative of (D.1), substituting (A.3), (22), (25), and time derivative of (C.2), results in

$$
\begin{aligned}
\dot{V}_{2} & =e^{T}(r-\Lambda e)+r^{T}\left[N_{d}+\widetilde{N}-e-\left[\Phi^{T}, 0\right]^{T}-r\right. \\
& -k_{g} r-\operatorname{diag}\left\{k_{d, 1}, k_{d, 2}, \ldots, k_{d, m-1}, 0\right\} r \\
& -D U D \beta \operatorname{Sgn}(e)+Y \widetilde{\phi}]-\widetilde{\phi}^{T} Y^{T} r \\
& -r^{T}\left[N_{d}-D U D \beta \operatorname{Sgn}(e)\right] .
\end{aligned}
$$

After cancelling same terms, utilizing (A.8) and (A.9), the right-hand side of (D.5) can be upper bounded as

$$
\dot{V}_{2} \leq-\left[\min \left\{\lambda_{\min }(\Lambda), 1\right\}-\frac{\rho^{2}}{4 k_{g}}-\sum_{i=1}^{m-1} \frac{\zeta_{\Phi_{i}}^{2}}{4 k_{d, i}}\right]\|z\|^{2}
$$

Provided that the control gains $\Lambda, k_{g}, k_{d, 1}, \ldots, k_{d, m-1}$ are selected sufficiently high, the below expression can be obtained

$$
\dot{V}_{2} \leq-c_{3}\|z\|^{2}
$$

where $c_{3}$ is some positive bounding constant. From (D.1) and (D.7), it is clear that $V_{2}(w)$ is non-increasing and bounded. After integrating (D.7), it can be stated that $z(t) \in \mathcal{L}_{2}$. Since $z(t) \in \mathcal{L}_{\infty} \cap \mathcal{L}_{2}$ and $\dot{z}(t) \in \mathcal{L}_{\infty}$, from Barbalat's Lemma [36], $\|z(t)\| \rightarrow 0$ as $t \rightarrow \infty$, thus meeting the control objective. Since no restrictions with respect to the initial conditions of the error signals were imposed on the control gains, the result is global.

\section{REFERENCES}

[1] F. Lewis and B. L. Stevens, "Aircraft Control \& Simulation," New York, NY, USA: John Wiley \& Sons, 2003.

[2] D. Enns, D. Bugajski, R. Hendrick, and G. Stein, "Dynamic inversion: An evolving methodology for flight control design," Int. J. of Control, vol. 59, no. 1, pp. 71-91, 1994.

[3] M. W. Oppenheimer and D. B. Doman, "Control of an unstable, nonminimum phase hypersonic vehicle model," IEEE Aerosp. Conf., Big Sky, MT, USA, 2006, pp. 1-7.

[4] D. Enns and T. Keviczky, "Dynamic inversion based flight control for autonomous RMAX helicopter," American Control Conf., Minneapolis, MN, USA, 2006, pp. 3916-3923.

[5] Z. Zhang, F. Hu, and J. Li, "Autonomous flight control system designed for small-scale helicopter based on approximate dynamic inversion," Int. Conf. on Adv. Comp. Cont., 2011, pp. 185-191. 
[6] Z. Liu, F. Zhou, and J. Zhou, "Flight control of unpowered flying vehicle based on robust dynamic inversion," Chinese Control Conf., Heilongjiang, China, 2006, pp. 693-698.

[7] T. Yamasaki, H. Sakaida, K. Enomoto, H. Takano, and Y. Baba, "Robust trajectory-tracking method for UAV guidance using proportional navigation," Int. Conf. on Cont. Autom. Sys., 2007, pp. 1404-1409.

[8] Q. Wang and R. F. Stengel, "Robust nonlinear flight control of a high performance aircraft," IEEE Tr. Control Sys. Tech., vol. 13, no. 1, pp. 15-26, 2005.

[9] Z. Xie, Y. Xia, and M. Fu, "Robust trajectorytracking method for UAV using nonlinear dynamic inversion," IEEE Int. Conf. Cyber. \& Intel. Sys., 2011, pp. 93-98.

[10] A. D. Ngo and D. B. Doman, "Dynamic inversionbased adaptive/reconfigurable control of the X-33 on ascent," IEEE Aerosp. Conf., Big Sky, MT, USA, 2006, pp. 2683-2697.

[11] M. D. Tandale and J. Valasek, "Adaptive dynamic inversion control of a linear scalar plant with constrained control inputs," American Control Conf., Portland, OR, USA, 2005, pp. 2064-2069.

[12] X. J. Liu, F. Lara-Rosano, and C. W. Chan, "Modelreference adaptive control based on neurofuzzy networks," IEEE Tr. Sys. Man Cyber. C, Appl. Rev., vol. 34, no. 3, pp. 302-309, 2004.

[13] J. Chen, D. Li, X. Jiang, and X. Sun, "Adaptive feedback linearization control of a flexible spacecraft," Conf. Intell. Sys. Design Appl., Jinan, China, 2006, pp. 225-230.

[14] Q. Wang and R. F. Stengel, "A dynamic inversion controller design for miniature Unmanned Aerial Vehicles," Consumer Elec., Comm. \& Networks, pp. 1916-1921, 2011.

[15] A. Calise and R. Rysdyk, "Nonlinear adaptive flight control using neural networks," IEEE Control Sys. Mag., vol. 18, no. 6, pp. 14-25, 1998.

[16] E. Lavretsky and N. Hovakimyan, "Adaptive compensation of control dependent modeling uncertainties using time-scale separation," IEEE Conf. on Decision $\&$ Control, Seville, Spain, 2005, pp. 2230-2235.

[17] J. Leitner, A. Calise, and J. V. R. Prasad, "Analysis of adaptive neural networks for helicopter flight controls," J. Guidance Control Dynamics, vol. 20, no. 5, pp. 972-979, 1997.

[18] Y. Shin, "Neural network based adaptive control for nonlinear dynamic regimes," PhD diss., Georgia Inst. Tech., Atlanta, GA, USA, 2005.

[19] E. N. Johnson and A. J. Calise, "Pseudo-control hedging: A new method for adaptive control," Workshop Adv. Guid. Cont. Tech., Redstone Arsenal, AL, USA, 2000.

[20] C. Schumacher and J. D. Johnson, "PI control of a tailless fighter aircraft with dynamic inversion and neural networks," American Control Conf., 1999, pp. 4173-4177.

[21] R. Rysdyk, F. Nardi, and A. J. Calise, "Robust adaptive nonlinear flight control applications using neural networks," American Control Conf., 1999, pp. 2595-2599.

[22] J. Shin, H. J. Kim, K. Youdan, and W. E. Dixon, "Asymptotic attitude tracking of the rotorcraft-based
UAV via RISE feedback and NN feedforward," IEEE Conf. Decision \& Control, 2010, pp. 3694-3699.

[23] J. Shin, H. J. Kim, K. Youdan, and W. E. Dixon, "Autonomous Flight of the Rotorcraft-Based UAV Using RISE Feedback and NN Feedforward Terms," IEEE Tr. Control Sys. Tech., vol. 20, no. 5, pp. 13921399, 2012.

[24] W. MacKunis, P. M. Patre, M. K. Kaizer, and W. E. Dixon, "Asymptotic Tracking for Aircraft via Robust and Adaptive Dynamic Inversion Methods," IEEE Tr. Control Sys. Tech., vol. 18, no. 6, pp. 1448-1456, 2010.

[25] B. Xian, D. M. Dawson, M. S. de Queiroz, and J. Chen, "A continuous asymptotic tracking control strategy for uncertain nonlinear systems," IEEE Tr. Automatic Control, vol. 49, no. 7, pp. 1206-1211, 2004.

[26] P. M. Patre, W. MacKunis, C. Makkar, and W. E. Dixon, "Asymptotic tracking for systems with structured and unstructured uncertainties," IEEE Conf. on Decision \& Control, 2006, pp. 441-446.

[27] L. Duan, W. Lu, F. Mora-Camino, and T. Miquel, "Flight-path tracking control of a transportation aircraft: Comparison of two nonlinear design approaches," Digit. Avi. Sys. Conf., Portland, OR, USA, 2006, pp. 1-9.

[28] I. Szaszi, B. Kulcsar, G. J. Balas, and J. Bokor, "Design of FDI filter for an aircraft control system," American Control Conf., 2002, pp. 4232-4237.

[29] W. MacKunis, "Nonlinear Control for Systems Containing Input Uncertainty via a Lyapunov-Based Approach," Ph.D. dissertation, University of Florida, Gainesville, FL, USA, 2009.

[30] R. R. Costa, L. Hsu, A. K. Imai, and P. Kokotovic, "Lyapunov-based adaptive control of MIMO systems," Automatica, vol. 39, no. 7, pp. 1251-1257, 2003.

[31] G. Tao, "Adaptive Control Design and Analysis," New York, NY, USA: John Wiley \& Sons, 2003.

[32] W. MacKunis, M. K. Kaiser, P. M. Patre, and W. E. Dixon, "Asymptotic tracking for aircraft via an uncertain dynamic inversion method," American Control Conf., Seattle, WA, USA, 2008, pp. 3482-3487.

[33] W. MacKunis, M. K. Kaiser, P. M. Patre, and W. E. Dixon, "Adaptive dynamic inversion for asymptotic tracking of an aircraft reference model," AIAA Guid. Nav. Cont. Conf., Honolulu, HI, USA, 2008, AIAA2008-6792.

[34] Dep. of Transportation, "Airworthiness Standards: Transport category airplanes," in Federal Aviation Regulations - Part 25, DC, USA, 1996.

[35] V. Stepanyan and A. Kurdila, "Asymptotic tracking of uncertain systems with continuous control using adaptive bounding," IEEE Tr. Neural Networks, vol. 20, no. 8, pp. 1320-1329, 2009.

[36] H. K. Khalil, "Nonlinear Systems," New York, NY, USA: Prentice Hall, 2002.

[37] B. Bidikli, E. Tatlicioglu, E. Zergeroglu, and A. Bayrak, "An Asymptotically Stable Continuous Robust Controller for a Class of Uncertain MIMO Nonlinear Systems," ArXiv e-prints:1301.5483, Jan. 2013. 